\title{
MARCELLA LEOPIZZI, Les sources documentaires du courant libertin français. Giulio Cesare Vanini
}

\section{Michele Mastroianni}

\section{(2) OpenEdition}

\section{Journals}

\section{Edizione digitale}

URL: http://journals.openedition.org/studifrancesi/33076

DOI: 10.4000/studifrancesi.33076

ISSN: 2421-5856

\section{Editore}

Rosenberg \& Sellier

\section{Edizione cartacea}

Data di pubblicazione: 1 décembre 2005

Paginazione: 631

ISSN: 0039-2944

\section{Notizia bibliografica digitale}

Michele Mastroianni, «MARcella Leopizzı, Les sources documentaires du courant libertin français. Giulio Cesare Vanini», Studi Francesi [Online], 147 (XLX | III) | 2005, online dal 01 novembre 2015, consultato il 18 avril 2021. URL: http://journals.openedition.org/studifrancesi/33076 ; DOI: https://doi.org/10.4000/ studifrancesi.33076 


\title{
MARCELLA LEOPIZZI, Les sources documentaires du courant libertin français. Giulio Cesare Vanini
}

\author{
Michele Mastroianni
}

\section{NOTIZIA}

MARCELLA LEOPIZZI, Les sources documentaires du courant libertin français. Giulio Cesare Vanini, préface de GIovanni Dotoli, Fasano (Bari)/Paris, Schena/Presse de l'Université de ParisSorbonne, 2004, pp. 823.

1 Il lavoro - di grande impegno - di Marcella Leopizzi (che esce quasi in contemporanea con quello di DIDIER FOUCAULT, Un philosophe libertin dans l'Europe baroque, Giulio Cesare Vanini, Paris, Champion, 2003) si propone, ripercorrendo l'aventure humaine, culturelle, libertine di Giulio Cesare Vanini, di studiarne il ruolo di trait-d'union tra la cultura italiana e la cultura europea nella fondazione e diffusione di un naturalismo che anticipa il razionalismo illuministico. Questo, dopo avere messo ordine in una mole enorme di dati in cui, alla rinfusa, si mescolavano documenti, tradizioni romanzesche e miti storiografici, insieme a veri e proprî errori. Come giustamente l'A. sottolinea, «nel corso dei quattro secoli che ci separano dalla nascita di Vanini occorre riconoscere che hanno regnato una profonda ignoranza e fraintendimenti riguardo la biografia e i testi di questo autore. L'utilizzazione erronea del suo nome, la ricostruzione sbagliata degli episodi della sua biografia - del suo luogo di nascita, delle tappe dei suoi viaggi, dei suoi studi, dei suoi incontri, dei suoi intrighi e delle sue fughe - l'affermazione ingannatrice secondo cui sarebbe stato condannato a causa delle sue opere, mentre in realtà il Parlamento di Tolosa, allorché emise la sua condanna a morte, non conosceva la sua identità e di conseguenza le sue pubblicazioni, così pure come le analisi imprecise dei suoi due libri, sono soltanto alcuni esempi degli errori commessi contro Vanini». Se una generazione più recente di storici ha compiuto studi seri e rigorosi nel ricostruire la 
vita e illuminare il pensiero di questo autore, che ora possiamo dire di conoscere al di là di una mitografia incrostata da secoli, restava per certo un aspetto importante da chiarire e da approfondire, quello dell'influenza esercitata all'interno del courant libertin francese. È questo il compito che intende svolgere M. Leopizzi, sfruttando anche quanto di valido hanno prodotto le ricerche esistenti su Vanini, per dimostrare la sua presenza nelle opere degli esprits forts, lungo tutto il Seicento, secolo in cui i suoi scritti, anche quando non sono citati espressamente, hanno rappresentato un serbatoio di temi e un modello di scrittura per i liberi pensatori; ricostruendone anzitutto la biografia per gli anni del soggiorno francese, sulla base di documenti finora sconosciuti o a malapena segnalati, ritrovati a Tolosa (in particolare le note manoscritte inedite di Adolphe Baudouin, archivista dell'Ottocento e studioso erudito di Vanini). Grande merito, appunto, di M. Leopizzi è di offrire una documentazione ricchissima di pièces a volte inedite, a volte già conosciute dagli specialisti, ma studiate in modo alquanto dispersivo, a volte segnalate dagli studiosi ma soltanto riassunte, mentre qui vengono trascritte per esteso. In apertura del lavoro abbiamo un'accurata cronologia, che si serve dei dati offerti da F. DE PAOLA, Giulio Cesare Vanini da Taurisano, filosofo europeo, Fasano, Schena, 1998. Nella prima parte (L'aventure humaine, pp. 45-453) viene ricostruito minuziosamente il soggiorno di Vanini in Francia, con ricchissima documentazione, come pure vengono presentate le reazioni dei contemporanei francesi, esponenti del potere politico e religioso (François de Rosset, Jacques Gaultier, François Garasse, Marin Mersenne, ecc.). La seconda parte (L'aventure culturelle, pp. 457-520) intende dimostrare che le due opere di Vanini - l'Amphitheatrum aeternae providentiae e il De admirandis naturae reginae deaeque mortalium arcanis - sono «due libri a carattere dia-logico che, basati sulla simulazione e sulla dissimulazione, contengono una dialogicità sostanziale, cioè un'interazione fra diversi punti di vista». E tuttavia più che risultati di un plagio sono risultati di una stratégie de collage atta a infondere un doppio senso nel discorso. La terza parte (L'aventure libertine, pp. 523-567) intende dimostrare che Vanini «ha gettato le basi della retorica sovversiva libertina che consiste nell'esposizione di idee trasgressive velate dalla simulazione del rispetto dei principi sino allora ammessi». La quarta parte infine (La présence de G. C. Vanini dans l'cuvre de quelques 'esprits forts', pp. 571-724) indaga la presenza di Vanini nel Theophrastus Redivivus, nei Trois imposteurs, in La Mothe Le Vayer, Naudé, Cyrano de Bergerac, Sorel e Bayle. Conclude il lavoro una ricca, e utilissima, bibliografia. 